\title{
Laser-Induced Forward Transfer-printing of pre-machined crystalline magneto-optic garnet discs
}

\author{
Collin L Sones, Matthias Feinaeugle, Behrad Gholipour, Alberto Sposito, Robert W Eason. \\ ${ }^{1}$ Optoelectronics Research Centre, University of Southampton, Southampton, SO17 1BJ, UK \\ Author(C.L. Sones) e-mail address: cls@orc.soton.ac.uk, Tel: (+44)02380 59 3141, Fax: (+44) 02380593142
}

\begin{abstract}
We present femtosecond laser-induced forward transfer of focussed ion beam premachined crystalline magneto-optic Yttrium Iron Garnet films. Debris-free circular micro-discs with smooth edges and surface uniformity have been successfully printed.

OCIS codes: (350.3390) Laser materials processing; (310.6845) Microstructure fabrication (220.4000)
\end{abstract}

\section{Introduction}

Laser-Induced Forward Transfer (LIFT) is a laser-based additive printing technique [1] which has been routinely employed for the printing of many different materials, on various substrates for applications as diverse as embedded electronic circuits to biosensors and tissue engineering. LIFT is essentially a single-shot, serial printing approach that allows the creation of a user-defined 1D/2D deposition pattern formed via a laser-assisted spatially controlled point-by-point pixelated deposition of the required material. This laser-based approach has been widely trialled for printing since it presents several advantages such as requiring a standard ambient environment for its implementation, providing the possibility of printing a wide range of materials, and allowing printing of multilayered stacks composed of dissimilar materials on both structured and planar substrates. Additionally, control of the size and shape of the printed pixels is achievable through the possibility of controlling the incident laser parameters such as wavelength, pulse-duration, and beam shape.

There are however several fundamental problems with LIFT printing which need to be addressed if precise and debris-free printing of high quality deposits is to be achieved. The first concerns the unavoidable partial ablation or melting of a surface region of the printed material (termed the donor) due to the absorption of the laser pulse responsible for the forward transfer process. Several variants to the basic LIFT process have been proposed and demonstrated by different research groups to alleviate this issue. These LIFT-variants are based on the use of an absorbing layer [2] sandwiched between the donor and the carrier substrate which thereby eliminates the direct interaction between the laser radiation and the donor. This sacrificial layer, which is often referred to as a dynamic release layer, decomposes into gaseous fragments upon absorption of the laser pulse, and provides the explosive push required to propel the donor towards the receiver. However, the use of a DRL for LIFT-transfer of comparatively brittle materials such as single crystals is problematic since the growth of a thin crystalline donor film directly on top of a polymer DRL is impossible because the high substrate temperatures of $\sim 600-800^{\circ} \mathrm{C}$ required for thin film epitaxial growth are far higher than the polymer decomposition temperature of $\sim 250^{\circ} \mathrm{C}$. A second problem concerns the unavoidable shearing and tearing of the donor around its edges during transfer. This poses an important constraint and sets a limit both for debris-free transfer and also for donors that are fragile, brittle, of substantial thickness ( $>$ a few $\mu \mathrm{m}$ ), or composite stacks where edge quality or layer mixing is an issue. A method of prepatterning is therefore highly desirable to precisely define the spatial extent of donor transfer to achieve much more precise and controlled printing results. A laser-based pre-indentation approach that uses preliminary laser pulses to define and weaken a boundary and a subsequent pulse to LIFT the donor has already been reported in the literature [3], however, the issue of non-uniform edge quality was not resolved.

In order to alleviate all of the above problems, we have earlier proposed and demonstrated [4] the use of a Focussed Ion Beam (FIB) to assist in partial pre-machining of the donor prior to LIFT. This pre-machining defines and weakens a boundary along which the pre-machined donor pixel can be easily ripped and propelled forward without undue shattering. We reported the successful LIFT-printing of intact amorphous ZnO pixels with extremely smooth edge quality and well-defined shapes via this route. In this contribution we present our initial results from the implementation of this approach to print debris-free, intact, crystalline Yttrium Iron Garnet (YIG) pixels.

\section{Experiments and Results}

The laser used for the LIFT-printing was a tunable ultrafast Ti:sapphire laser system operating at 800nm, with a repetition rate of $1 \mathrm{Khz}$, a pulse duration of $\sim 150 \mathrm{fs}$, and a maximum energy of $\sim 2 \mathrm{~mJ}$ per pulse. The laser beam was initially collimated and then homogenised using a refractive optical element to yield a spatially uniform top-hat intensity profile which was $\sim 6 \mathrm{~mm}$ in diameter. An aperture subsequently positioned in the path of the beam allowed selection of the shape and size of the incident laser beam which was imaged using a microscope objective onto the 
carrier-donor interface. The diameter of the imaged circular beam profile was set to $\sim 10 \mu \mathrm{m}$, and was adapted to match the diameter of the pre-machined donor. The crystalline YIG donor films were previously grown by pulsed laser deposition onto $10 \times 10 \times 1 \mathrm{~mm}^{3}$ Yttrium Aluminium garnet (YAG) carrier substrates. The YIG donor films were grown to have a thickness of $\sim 1 \mu \mathrm{m}$. YIG was chosen as the donor because its absorption was well matched with the two-photon absorption wavelength $(400 \mathrm{~nm})$ that would be required when LIFT-printing with fs-laser pulses. Our earlier attempts to LIFT crystalline Gadolinium Gallium Garnet (GGG) were unsuccessful because of the mismatched absorption criteria. The YIG-donor/YAG-carrier composite was then placed in near proximity to a Parafilm coated glass receiver for LIFT-printing. Carriers coated with Parafilm were used to ensure better adhesion of the LIFT-printed donor pixels. In order to maintain a uniform separation between the donor and the receiver a $1 \mu \mathrm{m}$ thick Mylar spacer was introduced between them. The incident laser fluences that were trialled for the LIFT printing ranged from $\sim 0.2-5.5 \mathrm{~J} / \mathrm{cm}^{2}$. Circular ring patterns that had outer and inner diameters of $\sim 10 \mu \mathrm{m}$ and $9.5 \mu \mathrm{m}$ respectively were pre-machined into the YIG donor films using a FIB. Arrays of rings were pre-machined to different depths (ranging from $40-80 \%$ of the initial thickness of the donor) to understand the dependence of the laser fluence on the LIFT-printing process. It is not necessary to machine throughout the entire depth of the donor as this would prevent the laser-induced pressure build-up behind the material intended for transfer.

Our first results for LIFT-printing from pre-machined and non-machined YIG donor films are shown in Fig.1. Fig. 1a shows a circular disc LIFT-printed from a pre-machined donor. The circular disc in Fig.1a appears to be slightly non-circular; however, this is because the front section of the LIFT-printed disc is slightly embedded in the soft Parafilm that covers the glass receiver. This also restricted our ability to image the side of this LIFT-printed pixel. Fig.1b thus shows an SEM image of the edge of another FIB pre-machined LIFT-printed pixel. For comparison, the SEM images of Fig.1c and Fig.1d show the top and side-view of an irregularly shaped, and partially fragmented pixel, LIFT-printed using a circular laser spot, but from a non-machined section of the donor. The undesirable and in our experience unavoidable shattering associated with pixels deposited with similar laser fluences, but without the essential pre-machining step is obvious. The SEM image of a non-machined LIFT-printed pixel in Fig.1d further emphasises the inevitable non-uniformity along its edge and in its thickness. Also seen in Fig.1d is a large semi-circular fragmented section (indicated by an arrow) from another non-machined LIFT-printed pixel. The SEM images of Fig.1b and Fig.1d that show side-on views were taken with the features tilted to $70^{\circ}$.

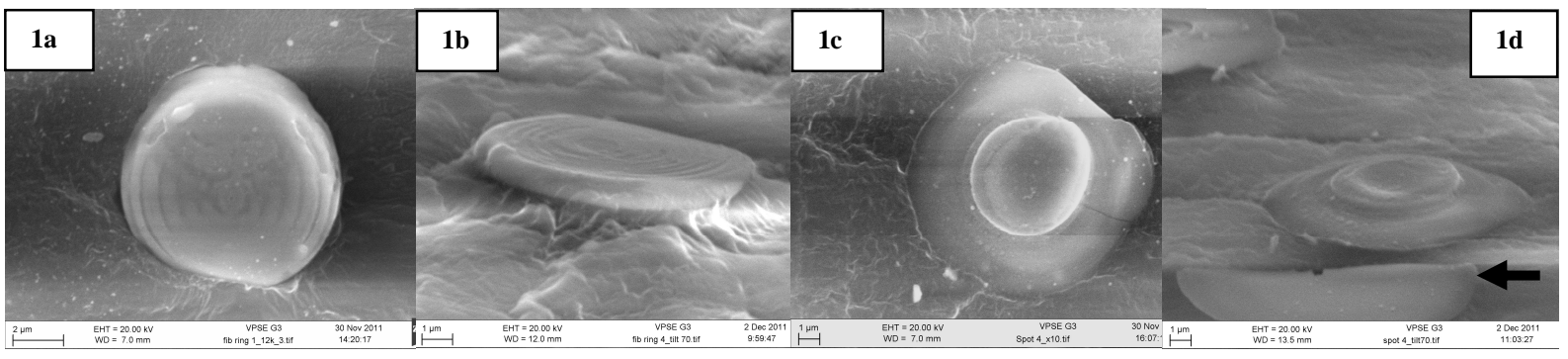

Fig.1: SEM images showing (a) the top-view and (b) the side-view of a FIB pre-machined LIFT-printed YIG pixel; SEM images showing (c) the top-view, and (d) the side-view of a non-machined LIFT-printed YIG pixel. Note that Fig.1d also shows fragmented pixel debris associated with typical non-machined LIFT-printing.

\section{Conclusions}

We have demonstrated successful debris-free, intact, uniformly shaped, LIFT-printing of FIB pre-machined crystalline YIG pixels with very good edge quality. To our knowledge this is the first report of LIFT-printing of intact crystalline materials from thin film donors, and we believe that this is an important step that will help spread the applicability of LIFT-printing for photonic single-crystal devices where edge quality and feature shape are crucial parameters.

\section{References}

[1] J. Bohandy, B.F. Kim, F.J. Adrian, "Metal deposition from a supported metal film using an excimer laser” J. Appl. Phys. 60,1538-39 (1986). [2] C. B. Arnold, P. Serra and A. Pique, "Laser direct-write techniques for printing of complex materials," MRS Bull. 32, 23-31 (2007).

[3] D.P.Banks, C.Grivas, I.Zergioti, R.W.Eason,"Ballistic laser-assisted solid transfer (BLAST) of intact material from a thin film precursor" Opt. Exp. 16(5), 3249-3254 (2008)

[4] K. S. Kaur, M. Feinaeugle, D.P. Banks, J.Y. Ou, F. Di Pietrantonio, E. Verona, C.L. Sones and R.W. Eason, "Laser-induced forward transfer of focussed ion beam pre-machined donors," Appl. Surf. Sci. 257, 6650-6653 (2011). 